Artículo

\title{
Productividad de higuerilla (Ricinus communis L.) en el norte de Sinaloa
}

\author{
Genny Llaven Valencia ${ }^{1 \S}$ \\ Alberto Borbon Gracia ${ }^{2}$ \\ Xochil Militza Ochoa Espinoza ${ }^{2}$ \\ Oralia Antuna Grijalva ${ }^{3}$ \\ Aidé Hernández Hernández ${ }^{3}$ \\ José Luis Coyac Rodríguez ${ }^{3}$
}

${ }^{1}$ Campo Experimental Valle Del Fuerte-INIFAP. Carretera Internacional México-Nogales km 1609, Col. Juan José Ríos, Guasave, Sinaloa, México. CP. 81110. ${ }^{2}$ Campo Experimental Norman E. BorlaugINIFAP. Calle Dr. Norman E. Borlaug km 12, Col. Valle del Yaqui, Cajame, Ciudad Obregón, Sonora. CP. 8500. (borbon.alberto@inifap.gob.mx; ochoa.xochil@inifap.gob.mx). ${ }^{3}$ Universidad Autónoma Agraria Antonio Narro. Periférico Raúl López Sánchez y carretera Santa Fe, Torreón, Coahuila, México. CP.27054. (oantuna_77@hotmail.com; jlcoyac@yahoo.com).

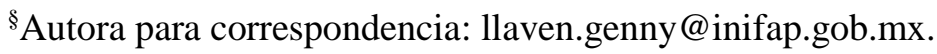

\section{Resumen}

Para determinar la productividad de higuerilla en Sinaloa, se evaluaron la influencia de dos fechas de siembra, disponibilidad de agua y dos densidades de siembra en el rendimiento de grano de cuatro híbridos de higuerilla, el ensayo se estableció en Campo Experimental Valle del Fuerte. Durante los ciclos agrícolas otoño-invierno con fecha de siembra del 10 de diciembre de 2015 y primavera-verano 2015-2016 con fecha de siembra del 18 de febrero de 2016. Se utilizó un diseño de bloques completos al azar con cuatro repeticiones, la parcela experimental fue de cuatro surcos de $20 \mathrm{~m}$ de largo, con una separación de $0.80 \mathrm{~m}$, equivalente a $64 \mathrm{~m}^{2}$, se manejaron

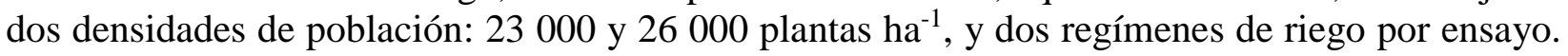
El análisis estadístico indicó que los híbridos 2B-5, Chinatan y HB-8, resultaron superiores en rendimiento y sin diferencias estadísticas, con días a madurez de 145 a 152, por lo que se consideran de ciclo normal; la altura promedio fue de $20 \mathrm{~m}$, considerada de porte medio. El ciclo que más favorece el desarrollo del cultivo para HB-8 y 2B-5 es O-I con densidad de 23000 plantas ha ${ }^{-1} \mathrm{y}$ tres riegos de auxilio para primavera-verano, se recomiendan cuatro riegos de auxilio, densidad de 23000 plantas ha ${ }^{-1}$ y los híbridos de mejor respuesta fueron HB-8 y Chinatan con rendimientos superior a $3000 \mathrm{~kg} \mathrm{ha}^{-1}$.

Palabras clave: ácidos grasos, biocombustible, estrés hídrico, índices de eficiencia, rendimiento.

Recibido: enero de 2019

Aceptado: mayo de 2019 


\section{Introducción}

La rentabilidad del cultivo de higuerilla depende de la planeación de las siembras, la diversificación y la pureza del material que se ofrezca al productor. Los costos de producción se incrementan por factores que afectan la rentabilidad; entre los principales destacan el combate de malezas y la cosecha manual o mecanizada, además de aspectos de manejo agronómico como elección inadecuada del terreno, fecha, densidad de siembra, uso y manejo eficiente del agua de riego, plagas y enfermedades, entre otros (Toledo et al., 2006; Rico et al., 2011).

Debido a la falta de diversidad de cultivos adaptados a las regiones de riego y temporal, se ha ocasionado la migración a las grandes ciudades en busca de alternativas de ingresos, por lo que es importante propiciar actividades agrícolas que generen empleos y permitan un aprovechamiento sustentable de los recursos fitogenéticos que pueden adaptarse a la región fortaleciendo la producción agrícola y ampliando las opciones de cultivos alternativos en México.

La productividad de un cultivo está determinada por su potencial genético y el impacto del ambiente sobre su capacidad de crecimiento y partición de materia seca hacia destinos reproductivos por otro lado los cambios en la fecha de siembra en cultivos bioenergéticos modifican la respuesta del rendimiento en grano y contenido de aceite. La temperatura es uno de los factores físicos de mayor importancia que influye directamente en el crecimiento y longitud de la planta durante su ciclo vegetativo (Msaakpa, 2014).

La higuerilla es un arbusto perenne tropical que se originó en África es una planta oleaginosa caracterizada por su rápido crecimiento y adaptación a diferentes zonas climáticas (Pabón, 2010), requiere de 140 a 180 días de estación de crecimiento presenta polinización natural cruzada, pues es una especie monoica (Chan et al., 2010). El principal producto del cultivo de la higuerilla es el grano de donde se extrae el aceite, también llamado aceite de ricino o (en inglés) y tiene numerosas aplicaciones industriales ya que es usado para la producción de plásticos, fibras sintéticas, tintas, esmaltes, lubricantes, cosmetología, química, fertilizantes, pesticidas, aeronáutica, médica, energética, entre otros (Cardona, 2009).

El grano de higuerilla tiene alto contenido de aceite, entre 45 y $55 \%$, comparado con el girasol que contiene entre el 38 y 48\%, la soya que tiene entre 40 y 47\% y el algodón que contiene entre el 15 y 19\%, característica que hace a la planta muy atractiva (Solís et al., 2011; Rafael y Alfredo, 2013) para la extracción de este producto. La higuerilla es considerada como materia prima en la producción de bioenergéticos y aceites industriales, la bioenergía es aquella energía generada por la biomasa o material de origen biológico que se encuentra sobre la superficie (Pabón, 2010; Goytia et al., 2011).

Actualmente es cultivada en muchas regiones tropicales y subtropicales de todo el mundo. La superficie cultivada de higuerilla a nivel mundial en el 2009 fue de 1473751 ha, con una producción total de 1499111 t de semilla. Los países más importantes son: India con 840000 ha, China 210000 ha, Brasil 159205 ha, Paraguay 11000 ha, con una producción de semilla de 1098000 t, 190000 t, 90384 t, 13000 t, respectivamente (FAOSTAT, 2011). México es un país con grandes reservas de petróleo también es un país donde la agricultura juega un papel muy importante en la generación de alimentos para la población y materias primas para la industria. 
Dentro del territorio nacional hay regiones con gran potencial para la producción de distintos cultivos útiles para la elaboración de biocombustibles tales como el biodiesel y el bioetanol. En México hay gran interés por validar rendimiento de higuerilla en diferentes entidades. La higuerilla se puede encontrar ampliamente distribuida de manera silvestre, al lado de la carretera, en bordos de drenes y canales de riego lo cual demuestra que esta especie está plenamente adaptada a las condiciones climatológicas del país (Machado et al., 2012).

Por su parte, Falasca et al. (2012) mencionaron que la higuerilla puede ser una alternativa de producción para los productores y el mercado energético, tomando en cuenta que es un cultivo que se adapta a condiciones de estrés hídrico bajo un sistema de uso eficiente de agua. Así también Rodríguez y Zamarripa (2011) indicaron una alta competitividad de la higuerilla para biocombustible en relación a los cultivos actuales en Oaxaca. Los estudios realizados sobre los componentes del rendimiento proporcionan una orientación para lograr una producción óptima, ya que estos son interdependientes y cambian en respuesta a las condiciones ambientales.

Las correlaciones negativas entre los componentes de rendimiento son muy frecuentes (Machado et al., 2009; Rafael et al., 2013; Rivera-Brenes y Hernández-López, 2016). Algunos autores señalan que para la selección de variantes altamente productivas de higuerilla se debe hacer énfasis en el número de racimos y peso de fruto, así como la longitud de los racimos y peso de semillas (Machado et al., 2009, 2014; Goytia et al., 2011).

Con el objetivo de evaluar el comportamiento agronómico de materiales élite de higuerilla en Morelos, obteniendo diferencias altamente significativas para todas las variables analizadas, por lo anterior el objetivo de este trabajo es determinar la productividad de higuerila (Ricinus communis L.), la influencia de dos fechas de siembra, densidad de plantas y la disponibilidad de agua en el rendimiento de grano, la cual genera información que puede servir de base para la selección de genotipos para el establecimiento de cultivos bioenergéticos de esta especie.

\section{Materiales y métodos}

\section{Localización del área de estudio}

La presente investigación se desarrolló en los ciclos agrícola otoño-invierno 2015-2016 y primavera-verano 2016, dos calendarios de riego y dos densidades de siembra, para cada ciclo. Se establecieron lotes experimentales en el Campo Experimental Valle del Fuerte-INIFAP, con localización geográfica a $25^{\circ} 46^{\prime} 492$ ' de latitud norte y $108^{\circ} 48^{\prime} 181$ ' de latitud oeste con una altitud de 4 msnm (www.inegi.org.mx). Su clima es generalmente húmedo cálido, y tiene una temperatura media anual de $25.9{ }^{\circ} \mathrm{C}$. Se registra una temperatura mínima anual de $18{ }^{\circ} \mathrm{C}$ y una máxima anual $33.9{ }^{\circ} \mathrm{C}$, siendo la temporada más calurosa la que va de mayo a octubre. En el período de referencia, la precipitación pluvial promedio es 383 milímetros anuales, siendo los meses más lluviosos de julio a octubre (http://www.weatherbase.com).

\section{Manejo del experimento}

Genotipos evaluados. Los híbridos utilizados fueron: Maravilla, Chinatan, HB-8, y 2B-5, más cuatro testigos comerciales: Olga, Eva, K-93 y Zoya (Cuadro 1), provenientes de China y Argentina, fueron sembradas y cosechadas manualmente en el Campo Experimental Valle del Fuerte (Guasave), durante los ciclos otoño-invierno 2015-2016 y primavera-verano 2016. 
Cuadro 1. Genotipos evaluados.

\begin{tabular}{cc}
\hline Tratamientos & testigos \\
\hline Maravilla & Olga \\
Chinatan & Eva \\
HB-8 & K-93 \\
2B-5 & Zoya \\
\hline
\end{tabular}

\section{Diseño experimental}

Se empleó un diseño factorial con cuatro repeticiones, con cuatro niveles de observación: genotipos, dos ciclos agrícolas, dos densidades de siembra y dos calendarios de riego.

\section{Variables evaluadas}

Las características de planta consideradas fueron altura de planta (AP, cm), días a floración (DF, días), fin de floración (FDF, días), días a madurez (DM, días), longitud de racimos de primera generación (LRG1), peso de racimos por generación (PRG, g), peso de mil semillas (PMS, g) y el rendimiento de grano (REND, $\mathrm{kg} \mathrm{ha}^{-1}$ ), como lo establece Goytia et al. (2011).

\section{Análisis de datos y modelo experimental}

Se realizó un análisis de varianza de un diseño factorial con arreglo en parcelas divididas utilizando el programa SASv9.4 (2015), y la prueba de Tukey $(p \leq 0.05)$ para la comparación de medias entre los genotipos.

\section{Resultados y discusión}

\section{Análisis factorial combinado}

Se encontró significancia en híbridos, riegos y fechas en siete variables de planta evaluadas, la interacción híbridos por fechas de lectura fue significativa para inicio de floración, fin de floración, días a madurez, peso de racimo en primera generación, longitud de racimo en primera generación y rendimiento (Cuadro 2).

De acuerdo al análisis de la información en relación a híbridos, densidad, riegos y fechas, interacciones $\mathrm{H} * \mathrm{~F}, \mathrm{D} * \mathrm{~F}$ o $\mathrm{R} * \mathrm{~F}$.

Se detectaron diferencias en siete variables de planta evaluadas, riego fue significativo para rendimiento y para fechas fue días a floración, fin de floración y madurez fisiológica de los híbridos evaluados, la interacción hibrido por fecha fue significativa para seis variables, de las cuales dos de ellas repercuten significativamente en el rendimiento como son longitud de racimo en $1^{\text {ra }}$ generación y peso de racimo en la $1^{\text {ra }}$ generación (LRG y PRG) (Cuadro 2). 
Cuadro 2. Relación entre fuentes de variación y características morfológicas de planta, cuadrados medios y significancia.

\begin{tabular}{lccccccccc}
\hline \multicolumn{1}{c}{ FV } & GL $\begin{array}{c}\text { Días a } \\
\text { floración }\end{array}$ & $\begin{array}{c}\text { Fin de } \\
\text { floración }\end{array}$ & $\begin{array}{c}\text { Días a } \\
\text { madurez }\end{array}$ & $\begin{array}{c}\text { Altura de } \\
\text { planta } \\
(\mathrm{cm})\end{array}$ & $\begin{array}{c}\text { Longitud } \\
\text { de racimo } \\
\text { en 1 } 1^{\text {ra }} \\
\text { generación }\end{array}$ & $\begin{array}{c}\text { Peso de } \\
\text { racimo en } 1^{\text {ra }} \\
\text { generación }\end{array}$ & $\begin{array}{c}\text { Peso de } \\
\text { mil } \\
\text { semillas }\end{array}$ & $\begin{array}{c}\text { Rendimiento } \\
\left(\mathrm{kg} \mathrm{ha}^{-1}\right)\end{array}$ \\
\hline Híbrido & 7 & $1922.09^{* *}$ & $1691.6^{* *}$ & $1224.02^{* *}$ & $30264.1^{* *}$ & $1400.86^{* *}$ & $3259829.45^{* *}$ & 83985.9 & $2728043.73^{* *}$ \\
Densidad & 1 & 4.5 & 1.32 & 10.12 & 17.25 & 52.53 & 80601.12 & 532.19 & 164953.32 \\
Riegos & 1 & 3.12 & 1.75 & 1.53 & 14770.5 & 603.78 & 1637145.12 & 736.32 & $5204747.82^{* *}$ \\
fechas & 1 & $604^{* *}$ & $779.65^{* *}$ & $1714.54^{* *}$ & 7146.61 & 240 & 2464706.45 & 296.38 & 653133.65 \\
Error & 116 & 3.46 & 4.37 & 1.56 & 1178.02 & 82.6 & 324750.71 & 251.89 & 286135.6 \\
$\mathrm{H} * \mathrm{~F}$ & 7 & $73.32^{* *}$ & $22.78^{* *}$ & $231.13^{* *}$ & 5133.59 & $490.83^{* *}$ & $2538458.5^{* *}$ & $1224.561388594 .89^{* *}$ \\
$\mathrm{D} * \mathrm{~F}$ & 1 & 0.5 & 9.57 & 10.12 & 1960.94 & 30.03 & 3070861.53 & 815.07 & 32416.95 \\
$\mathrm{R} * \mathrm{~F}$ & 1 & 6.125 & 1.75 & 1.53 & 297 & 205.03 & 629161.53 & 689.13 & 2732829.76 \\
$\mathrm{CV}$ & & 2.55 & 2.34 & 0.81 & 13.97 & 14.43 & 23.1 & 4.12 & 18.06 \\
$\mathrm{R}^{2}$ & & 0.97 & 0.97 & 0.99 & 0.69 & 0.64 & 0.58 & 0.95 & 0.68 \\
\hline
\end{tabular}

${ }^{* *}=$ Diferencias significativas $\mathrm{a} \leq 0.05$ de probabilidad.

\section{Variación de características morfológicas de planta entre híbridos}

En cuanto a días a floración de los híbridos, 2-B5, Chinatan y HB-8 fueron más precoz con días a floración que van de 64 a 69, días respectivamente. De hecho, la diferencia de días desde el inicio de floración hasta la cosecha fue de 76 días tanto para Maravilla como para HB-8, y de 88 para 2B-5. Para Chinatan fue de 84 días, lo que indica que HB-8 tiene una mayor tasa de llenado de grano (TLLG, mayor acumulación de gramos por día, Maravilla $=34$ g día y HB-8= 42 g día. 2B$5=37 \mathrm{~g}$ día), podría considerarse que este material es precoz, dado que la precocidad es una característica relativa y es necesario considerarlo dentro del ciclo de producción, en lugares donde el ciclo de cultivo normal va de 140 a 180 días para los cultivos que se establecen en la zona, este material muestra ser de porte mediano con una media de dos metros de longitud y un ciclo de 140 días, su rendimiento promedio es de $3205 \mathrm{~kg} \mathrm{ha}^{-1}$.

También se observa claramente la división en dos grupos de acuerdo a su precocidad a los días a madurez en donde se muestra lo siguiente: ciclo precoz (138-143) ciclo medio (151-166). Para altura de planta se clasifican en porte bajo para los testigos, Olga, Eva, K-93 y Zoya (100-162 $\mathrm{cm}$ ), porte medio con altura que va de 230 a 236 para los híbridos HB-8, Chinatan y 2B-5. La de porte alto fue Maravilla con $309 \mathrm{~cm}$ de altura, cuya característica complico las labores de toma de dato y corte (Cuadro 3).

Las variables involucradas con el rendimiento como el peso de frutos del primer racimo o generación uno, longitud del primer racimo y peso de mil semillas mostraron valores que reflejan el potencial de los materiales 2B-5 y HB-8 los cuales respondieron con rendimientos favorables en ambos ciclos evaluados, las densidades y calendarios de riego en estudio fueron relevantes para identificar su adaptabilidad al someterlos a estrés hídrico y mayor competencia de plantas. 
En algunos estudios, el número de semillas por racimo se descompone en número de frutos por racimo y el número de semillas por fruto; sin embargo, estos dos componentes se analizan preferiblemente juntos porque hay una variación insignificante en el número de semillas por fruto (Machado et al., 2009). El peso individual de las semillas juega un papel importante, reportaron rendimientos de $3569 \mathrm{~kg} \mathrm{ha}^{-1}$ en temporal para la región de Durango.

Por su parte Machado et al. (2009) reportaron de 34 a 143 frutos por racimo. En Morelos encontraron rangos de días a floración de 40 a 72 dds. Los resultados encontrados en esta investigación fueron, rangos de DF fluctuaron de 99 a 149 dds, las diferencias residen en el nivel altitudinal y clima, siendo Morelos de clima más cálido y seco (Cuadro 3).

Cuadro 3. Comparación de medias de las características morfológicas de ocho híbridos de higuerilla.

\begin{tabular}{|c|c|c|c|c|c|c|c|c|}
\hline Híbrido & $\begin{array}{l}\text { Días a } \\
\text { floración }\end{array}$ & $\begin{array}{l}\text { Fin de } \\
\text { floración }\end{array}$ & $\begin{array}{c}\text { Días a } \\
\text { madurez }\end{array}$ & $\begin{array}{l}\text { Altura } \\
\text { de } \\
\text { planta } \\
(\mathrm{cm})\end{array}$ & $\begin{array}{c}\text { Longitud de } \\
\text { racimo en } \\
1^{\text {ra }} \\
\text { generación }\end{array}$ & $\begin{array}{c}\text { Peso de } \\
\text { racimo en } \\
1^{\text {ra }} \\
\text { generación }\end{array}$ & $\begin{array}{c}\text { Peso de } \\
\text { mil } \\
\text { semillas }\end{array}$ & $\begin{array}{l}\text { Rendimiento } \\
\qquad\left(\mathrm{kg} \mathrm{ha}^{-1}\right)\end{array}$ \\
\hline Maravilla & $90^{\mathrm{a}}$ & $106^{\mathrm{a}}$ & $166^{\mathrm{a}}$ & $309^{a}$ & $48^{\mathrm{bc}}$ & $3010^{\mathrm{a}}$ & $496^{a}$ & $2615^{\mathrm{ab}}$ \\
\hline $2 B-5$ & $64^{\mathrm{e}}$ & $82^{\mathrm{d}}$ & $152^{\mathrm{b}}$ & $236^{\mathrm{ab}}$ & $58^{\mathrm{abc}}$ & $2109^{a b}$ & $374^{\mathrm{b}}$ & $3249^{a}$ \\
\hline HB-8 & $69^{d}$ & $84^{\mathrm{cd}}$ & $145^{\mathrm{c}}$ & $230^{\mathrm{bc}}$ & $70^{\mathrm{a}}$ & $2656^{\mathrm{ab}}$ & $340^{\mathrm{cb}}$ & $3205^{\mathrm{ab}}$ \\
\hline Chinatan & $67^{\mathrm{d}}$ & $86^{\mathrm{cd}}$ & $151^{\mathrm{b}}$ & $235^{\mathrm{ab}}$ & $73^{\mathrm{ab}}$ & $2326^{\mathrm{ab}}$ & $352^{\mathrm{b}}$ & $3028^{\mathrm{ab}}$ \\
\hline Olga & $83^{b}$ & $92^{b}$ & $138^{\mathrm{d}}$ & $137^{\mathrm{d}}$ & $43^{b c}$ & $1523^{\mathrm{bc}}$ & $315^{\mathrm{cd}}$ & $2025^{\mathrm{b}}$ \\
\hline Eva & $78^{\mathrm{c}}$ & $86^{\mathrm{cd}}$ & $143^{c}$ & $157^{\mathrm{cd}}$ & $61^{\mathrm{ab}}$ & $2232^{\mathrm{ab}}$ & $243^{b c}$ & $2234^{\mathrm{ab}}$ \\
\hline K-93 & $69^{\mathrm{de}}$ & $76^{\mathrm{e}}$ & $138^{\mathrm{d}}$ & $162^{\mathrm{cd}}$ & $40^{\mathrm{c}}$ & $1790^{\mathrm{abc}}$ & $296^{d}$ & $2909^{\mathrm{ab}}$ \\
\hline Zoya & $81^{\mathrm{b}}$ & $88^{b c}$ & $138^{\mathrm{d}}$ & $100^{d}$ & $46^{\mathrm{bc}}$ & $567^{\mathrm{c}}$ & $196^{\mathrm{e}}$ & $629^{c}$ \\
\hline Media & 75 & 88 & 146 & 208 & 55 & 200 & 327 & 2487 \\
\hline $\mathrm{CV}$ & 2.55 & 2.34 & 0.81 & 13.97 & 14.43 & 23.1 & 4.12 & 18.06 \\
\hline DMS & 27.97 & 18 & 17.22 & 7722 & 20.45 & 1282.2 & 3571 & 1203.6 \\
\hline
\end{tabular}

\section{Variación entre híbridos por fechas en análisis combinado}

En relación con la cantidad de frutos (CFR) y granos en cada racimo (CGR), se observó una tendencia similar en primera generación de racimos; por tanto, esta correlación es consecuencia a que, cada fruto contiene tres carpelos que pueden originar igual número de granos; sin embargo, factores sanitarios, genéticos y ambientales, pueden regular, la cantidad final de grano por racimo. (Falasca et al., 2012).

De acuerdo con los trabajos realizados de Amorim et al. (2001); Moshkin (1986 ${ }^{\text {a }}$ y Weiss (1983), condiciones extremas, de frío $\left(<14{ }^{\circ} \mathrm{C}\right)$ y calor $\left(>41{ }^{\circ} \mathrm{C}\right)$, limitan la formación y maduración de las semillas, debido al aborto de flores y la reversión sexual, al incrementar la relación de flores masculinas sobre las femeninas. Sin embargo, los resultados obtenidos, indican que, en las condiciones donde se realizó la investigación y para las variedades evaluadas, las temperaturas máximas y mínimas del ambiente, no afectaron la formación ni el llenado del grano, conforme lo discutido anteriormente. El ciclo que más favorece el desarrollo del cultivo para HB8 y 2B-5 es O-I con densidad de 23000 plantas ha ${ }^{-1}$ y tres riegos de auxilio (Cuadro 4). 
Cuadro 4. Relaciones entre fuentes de variación y características de planta en el CEVAF.

\begin{tabular}{lcccccccc}
\hline Ciclo & $\begin{array}{c}\text { Días a } \\
\text { floración }\end{array}$ & $\begin{array}{c}\text { Fin de } \\
\text { floración }\end{array}$ & $\begin{array}{c}\text { Días a } \\
\text { madurez }\end{array}$ & $\begin{array}{c}\text { Altura } \\
\text { de } \\
\text { planta } \\
(\mathrm{cm})\end{array}$ & $\begin{array}{c}\text { Peso de } \\
\text { racimo en } \\
1^{\text {ra }} \\
\text { generación }\end{array}$ & $\begin{array}{c}\text { Longitud } \\
\text { de racimo } \\
\text { en } 1^{\text {ra }} \\
\text { generación }\end{array}$ & $\begin{array}{c}\text { Peso de } \\
\text { mil } \\
\text { semillas }\end{array}$ & $\begin{array}{c}\text { Rendimiento } \\
\left(\mathrm{kg} \mathrm{ha}^{-1}\right)\end{array}$ \\
\hline $1^{\text {er }}$ FS & 78 & 95 & 162 & 258 & 2259 & 54 & 389 & 3449 \\
23000 & $78^{\mathrm{a}}$ & $94^{\mathrm{b}}$ & $161^{\mathrm{b}}$ & $248^{\mathrm{b}}$ & $2324^{\mathrm{a}}$ & $53^{\mathrm{a}}$ & $400^{\mathrm{a}}$ & $3494^{\mathrm{a}}$ \\
26000 & $78^{\mathrm{b}}$ & $96^{\mathrm{a}}$ & $163^{\mathrm{a}}$ & $268^{\mathrm{a}}$ & $2185^{\mathrm{a}}$ & $54^{\mathrm{a}}$ & $378^{\mathrm{b}}$ & $3409^{\mathrm{a}}$ \\
3 R. AUX & $77^{\mathrm{a}}$ & $95^{\mathrm{b}}$ & $161^{\mathrm{b}}$ & $244^{\mathrm{b}}$ & $2170^{\mathrm{a}}$ & $52^{\mathrm{a}}$ & $378^{\mathrm{b}}$ & $3314^{\mathrm{b}}$ \\
4 R. AUX & $78^{\mathrm{b}}$ & $95^{\mathrm{a}}$ & $163^{\mathrm{a}}$ & $273^{\mathrm{a}}$ & $2358^{\mathrm{a}}$ & $55^{\mathrm{a}}$ & $401^{\mathrm{a}}$ & $3601^{\mathrm{a}}$ \\
promedio & 78 & 95 & 162 & 258 & 2259 & 54 & 389.2 & 3453 \\
$2^{\text {da }}$ FS & 68 & 83 & 143 & 233 & 64 & 2674 & 380 & 2473 \\
23 000 & $67^{\mathrm{a}}$ & $83^{\mathrm{a}}$ & $141^{\mathrm{b}}$ & $230^{\mathrm{a}}$ & $63^{\mathrm{a}}$ & $2459^{\mathrm{a}}$ & $376^{\mathrm{b}}$ & $2464^{\mathrm{a}}$ \\
26 000 & $67^{\mathrm{a}}$ & $82^{\mathrm{a}}$ & $143^{\mathrm{a}}$ & $237^{\mathrm{b}}$ & $63^{\mathrm{a}}$ & $2914^{\mathrm{b}}$ & $384^{\mathrm{a}}$ & $2482^{\mathrm{a}}$ \\
3 R. AUX & $67^{\mathrm{a}}$ & $83^{\mathrm{a}}$ & $141^{\mathrm{b}}$ & $216^{\mathrm{b}}$ & $59^{\mathrm{a}}$ & $2456^{\mathrm{b}}$ & $375^{\mathrm{b}}$ & $2137^{\mathrm{b}}$ \\
4 R. AUX & $67^{\mathrm{a}}$ & $82^{\mathrm{a}}$ & $143^{\mathrm{a}}$ & $252^{\mathrm{a}}$ & $68^{\mathrm{a}}$ & $2918^{\mathrm{a}}$ & $384^{\mathrm{a}}$ & $2850^{\mathrm{a}}$ \\
Promedio & 67 & 83 & 142 & 234 & 63 & 2684 & 380 & 2481 \\
\hline
\end{tabular}

Cuadrados medios y significancia, de dos fechas de siembra otoño-invierno y primavera verano de 2015-2016.

\section{Variación entre riegos por fechas}

Los materiales evaluados se comportaron de manera favorable a los calendarios de riego aplicados, puesto que se mantuvieron con rendimientos arriba de $3000 \mathrm{~kg} \mathrm{ha}^{-1}$, los testigos comerciales por su parte el más alto en rendimiento fue OLGA con $2681 \mathrm{~kg} \mathrm{ha}^{-1}$ y el de menor rendimiento ZOYA con $359 \mathrm{~kg} \mathrm{ha}^{-1}$.

\section{Densidad}

Se encontró variabilidad en los híbridos de higuerilla, en cuanto a las características morfológicas de planta, así como en altura de planta, inicio de floración, madurez fisiológica y sobre todo en rendimiento. Esto permitió diferenciar grupos, los cuales presentaron diferentes potenciales de producción superando a los testigos involucrados; de acuerdo con la altura de la planta, Silva (2005) agrupó la higuerilla en tres categorías: plantas de porte bajo $(<1.5 \mathrm{~m})$, medio $(1.5-2.5 \mathrm{~m}) \mathrm{y}$ alto $(>2.5 \mathrm{~m})$ por tanto, de acuerdo con los resultados logrados en esta investigación se observó que la altura en el lote experimental con baja densidad (23000 plantas ha ${ }^{-1}$ ) el rango de altura fue mucho más amplio donde está oscilo entre 113 a $268 \mathrm{~cm}$ de longitud; sin embargo, al incrementar la densidad a (26000 plantas ha ${ }^{-1}$ ) esta se redujo a un rango menor que van de $201 \mathrm{a} 302 \mathrm{~cm}$ de altura (Figura 1), no mostró efectos significativo en rendimiento puesto que a mayor densidad favorece competencia por luz, nutrientes; es decir, reduce la acumulación de biomasa en los órganos vegetativos disminuyendo la posibilidad de nutrientes para abastecer la demanda generada por las espigas, capsulas y granos en crecimiento (De Souza et al., 2011).

La altura del vegetal es importante, ya que de acuerdo con Weiss (1983), la cosecha manual o mecánica se dificulta, en la medida que los racimos de órdenes superiores sean emitidos a alturas mayores. 
Resultados similares fueron encontrados por Beltrão et al. (2001), quienes hallaron, al comparar dos localidades con diferente nivel altitudinal ( 27 y $500 \mathrm{msnm}$ ), que a medida que se incrementó la altura sobre el nivel del mar, las variedades evaluadas presentaron mayor porte o tamaño de la planta. De acuerdo a lo indicado por Tavora (1982); Weiss (1983); Moshkin (1986a); Amorim et al. (2001); Falasca et al. (2012), sobre el efecto de la temperatura y la altitud límite para el desarrollo de la planta y producción de semilla, los resultados obtenidos en el presente trabajo, evidenciaron que la planta de higuerilla se adaptó a rangos térmicos por debajo y por encima de los que, tradicionalmente, se han considerado propicios para su cultivo; asimismo, en relación con la elevación altitudinal, es evidente que, la higuerilla puede ser sembrada hasta $2.12 \mathrm{msnm}$, con el uso de materiales adaptados a ese ambiente, sin que se afecte su rendimiento.

\section{Análisis por ciclo agrícola}

\section{Primera fecha de siembra otoño-invierno 2015-2016}

En el Cuadro 5 y 6 se observa alta significancia entre híbridos para todos los caracteres evaluados; sin embargo, para la interacción híbridos por densidad únicamente se observó diferencia en rendimiento, que indica algunos híbridos a verse afectado por la alta densidad.

Cuadro 5. Relaciones entre fuentes de variación y características morfológicas de la planta del CEVAF.

\begin{tabular}{|c|c|c|c|c|c|c|c|c|c|}
\hline $\mathrm{FV}$ & GL & $\begin{array}{l}\text { Días a } \\
\text { floración }\end{array}$ & $\begin{array}{l}\text { Fin de } \\
\text { floración }\end{array}$ & $\begin{array}{l}\text { Días a } \\
\text { madurez }\end{array}$ & $\begin{array}{l}\text { Altura de } \\
\text { la planta } \\
(\mathrm{cm})\end{array}$ & $\begin{array}{l}\text { Peso de } \\
\text { racimo en } 1^{\text {ra }} \\
\text { generación }\end{array}$ & $\begin{array}{l}\text { Longitud } \\
\text { de racimo } \\
\text { en } 1^{\text {ra }} \\
\text { generación }\end{array}$ & $\begin{array}{c}\text { Peso de } \\
\text { mil } \\
\text { semillas }\end{array}$ & $\begin{array}{l}\text { Rendimiento } \\
\left(\mathrm{kg} \mathrm{ha}^{-1}\right)\end{array}$ \\
\hline Rep. & 3 & 0.291667 & 0 & 0 & 1878.7 & 225659.81 & 56.02 & 6727 & 84188.8 \\
\hline Densidad & 1 & 1 & 9 & 0 & 1173.06 & 10782222.64 & 1.56 & 1332.2 & 171810.25 \\
\hline Riegos & 1 & 0.25 & 0 & 0 & 5439.06 & 118250.02 & 52.56 & 1425.06 & 197358.06 \\
\hline Híbridos & 7 & $1276.23^{* *}$ & $997.9^{* *}$ & $1191.3^{* *}$ & $24984.3^{\text {*** }}$ & $5090182.96^{* *}$ & $612.1^{* *}$ & $46913.9^{* *}$ & $1996726.19^{* * *}$ \\
\hline $\begin{array}{l}\text { Híbridos } \\
\text { /densidad }\end{array}$ & 3 & 1.8 & 9 & 0 & 7 & 544400.8 & 185.85 & 309.6 & $233533.04^{* *}$ \\
\hline $\mathrm{CV}$ & & 0.4 & 1 & 1 & 11.72 & 26.8 & 19.5 & 3.9 & 15.7 \\
\hline $\mathrm{R}^{2}$ & & 0.9 & 0 & 0 & 0.81 & 0.67 & 0.47 & 0.96 & 0.52 \\
\hline
\end{tabular}

Cuadrados medios y significancia, primera fecha de siembra otoño-invierno de 2015-2016.

Cuadro 6. Relaciones entre fuentes de variación y características de planta en el CEVAF.

\begin{tabular}{lcccccccc}
\hline Ciclo & $\begin{array}{c}\text { Días a } \\
\text { floración }\end{array}$ & $\begin{array}{c}\text { Fin de } \\
\text { floración }\end{array}$ & $\begin{array}{c}\text { Días a } \\
\text { madurez }\end{array}$ & $\begin{array}{c}\text { Altura de } \\
\text { la planta } \\
(\mathrm{cm})\end{array}$ & $\begin{array}{c}\text { Peso de } \\
\text { racimo en } 1^{\text {ra }} \\
\text { generación }\end{array}$ & $\begin{array}{c}\text { Longitud de } \\
\text { racimo en } 1^{\text {ra }} \\
\text { generación }\end{array}$ & $\begin{array}{c}\text { Peso de } \\
\text { mil } \\
\text { semillas }\end{array}$ & $\begin{array}{c}\text { Rendimiento } \\
\left(\mathrm{kg} \mathrm{ha}^{-1}\right)\end{array}$ \\
\hline $1^{\text {er } F S}$ & 78 & 95 & 162 & 258 & 2259 & 54 & 389 & 3449 \\
23000 & $78^{\mathrm{a}}$ & $94^{\mathrm{b}}$ & $161^{\mathrm{b}}$ & $248^{\mathrm{b}}$ & $2324^{\mathrm{a}}$ & $53^{\mathrm{a}}$ & $400^{\mathrm{a}}$ & $3494^{\mathrm{a}}$ \\
26000 & $78^{\mathrm{b}}$ & $96^{\mathrm{a}}$ & $163^{\mathrm{a}}$ & $268^{\mathrm{a}}$ & $2185^{\mathrm{a}}$ & $54^{\mathrm{a}}$ & $378^{\mathrm{b}}$ & $3409^{\mathrm{a}}$ \\
3 R. AUX & $77^{\mathrm{a}}$ & $95^{\mathrm{b}}$ & $161^{\mathrm{b}}$ & $244^{\mathrm{b}}$ & $2170^{\mathrm{a}}$ & $52^{\mathrm{a}}$ & $378^{\mathrm{b}}$ & $3314^{\mathrm{b}}$ \\
4 R. AUX & $78^{\mathrm{b}}$ & $95^{\mathrm{a}}$ & $163^{\mathrm{a}}$ & $273^{\mathrm{a}}$ & $2358^{\mathrm{a}}$ & $55^{\mathrm{a}}$ & $401^{\mathrm{a}}$ & $3601^{\mathrm{a}}$ \\
Promedio & 78 & 95 & 162 & 258 & 2259 & 54 & 389.2 & 3453 \\
\hline
\end{tabular}

Cuadrados medios y significancia, primera fecha de siembra otoño-invierno de 2015-2016. 
En la comparación de medias para este ciclo se observa que los híbridos HB-8 y 2B-5 resultaron con mayor rendimiento en este ciclo superior $3000 \mathrm{~kg} \mathrm{ha}^{-1}$, el testigo más sobresaliente es Olga con rendimiento de $2681 \mathrm{~kg} \mathrm{ha}^{-1}$. Con relación a la evaluación de altura de planta, se aprecia que existen variaciones entre híbridos, la mayor altura observada es en el hibrido Maravilla con 347 $\mathrm{cm}$, mientras que de altura media son HB-8, Chinatan y 2B-5 la cual oscila entre 229 a $239 \mathrm{~cm}$, sin embargo, los cuatro testigos se mantuvieron dentro de porte bajo con alturas de 107, 160, 177 y $184 \mathrm{~cm}$ (Cuadro 7, 8 y 9).

Cuadro 7. Comparación de medias de las características morfológicas de planta e índice de selección de las variedades de higuerilla en el CEVAF.

\begin{tabular}{lcccccccc}
\hline Híbrido & $\begin{array}{c}\text { Días a } \\
\text { floración }\end{array}$ & $\begin{array}{c}\text { Fin de } \\
\text { floración }\end{array}$ & $\begin{array}{c}\text { Días a } \\
\text { madurez }\end{array}$ & $\begin{array}{c}\text { Altura } \\
\text { de } \\
\text { planta } \\
(\mathrm{cm})\end{array}$ & $\begin{array}{c}\text { Longitud } \\
\text { de racimo } \\
\text { en } 1^{\text {ra }} \\
\text { generación }\end{array}$ & $\begin{array}{c}\text { Peso de } \\
\text { racimo en } \\
1^{\text {ra }} \\
\text { generación }\end{array}$ & $\begin{array}{c}\text { Peso de } \\
\text { mil } \\
\text { semillas }\end{array}$ & $\begin{array}{c}\text { Rendimiento } \\
\left(\mathrm{kg} \mathrm{ha}^{-1}\right)\end{array}$ \\
\hline Maravilla & $97^{\mathrm{a}}$ & $113^{\mathrm{a}}$ & $180^{\mathrm{a}}$ & $347^{\mathrm{a}}$ & $49^{\mathrm{a}}$ & $3226^{\mathrm{a}}$ & $504^{\mathrm{a}}$ & $3349^{\mathrm{ab}}$ \\
T. Eva & $86^{\mathrm{b}}$ & $92^{\mathrm{b}}$ & $145^{\mathrm{d}}$ & $177^{\mathrm{ab}}$ & $63^{\mathrm{a}}$ & $3511^{\mathrm{a}}$ & $344^{\mathrm{b}}$ & $2669^{\mathrm{ab}}$ \\
T. Zoya & $86^{\mathrm{b}}$ & $92^{\mathrm{b}}$ & $145^{\mathrm{d}}$ & $107^{\mathrm{b}}$ & $49^{\mathrm{a}}$ & $81^{\mathrm{a}}$ & $135^{\mathrm{c}}$ & $359^{\mathrm{b}}$ \\
T. Olga & $85^{\mathrm{b}}$ & $92^{\mathrm{b}}$ & $145^{\mathrm{d}}$ & $160^{\mathrm{b}}$ & $45^{\mathrm{a}}$ & $1154^{\mathrm{a}}$ & $306^{\mathrm{b}}$ & $2681^{\mathrm{ab}}$ \\
HB-8 & $75^{\mathrm{c}}$ & $90^{\mathrm{b}}$ & $150^{\mathrm{c}}$ & $239^{\mathrm{ab}}$ & $45^{\mathrm{a}}$ & $2539^{\mathrm{a}}$ & $346^{\mathrm{b}}$ & $3782^{\mathrm{a}}$ \\
T. K-93 & $72^{\mathrm{c}}$ & $80^{\mathrm{b}}$ & $145^{\mathrm{d}}$ & $184^{\mathrm{ab}}$ & $32^{\mathrm{a}}$ & $685^{\mathrm{a}}$ & $294^{\mathrm{b}}$ & $1894^{\mathrm{ab}}$ \\
Chinatan & $70^{\mathrm{c}}$ & $92^{\mathrm{b}}$ & $162^{\mathrm{b}}$ & $239^{\mathrm{ab}}$ & $56^{\mathrm{a}}$ & $1971^{\mathrm{a}}$ & $358^{\mathrm{b}}$ & $3406^{\mathrm{ab}}$ \\
2B-5 & $68^{\mathrm{c}}$ & $88^{\mathrm{b}}$ & $162^{\mathrm{b}}$ & $229^{\mathrm{ab}}$ & $45^{\mathrm{a}}$ & $1522^{\mathrm{a}}$ & $382^{\mathrm{b}}$ & $3647^{\mathrm{a}}$ \\
Promedio & 80 & 92 & 154 & 263 & 48 & 1836 & 334 & 2723 \\
\hline
\end{tabular}

Primera fecha de siembra otoño-invierno de 2015-2016. Segunda fecha de siembra primavera-verano 2016.

Cuadro 8. Relaciones entre fuentes de variación y características morfológicas de planta en el CEVAF.

\begin{tabular}{lccccccccc}
\hline FV & GL & $\begin{array}{c}\text { Días a } \\
\text { floración }\end{array}$ & $\begin{array}{c}\text { Fin de } \\
\text { floración }\end{array}$ & $\begin{array}{c}\text { Días a } \\
\text { madurez }\end{array}$ & $\begin{array}{c}\text { Altura de } \\
\text { planta } \\
(\mathrm{cm})\end{array}$ & $\begin{array}{c}\text { Longitud } \\
\text { de racimo } \\
\text { en } 1^{\text {ra }} \\
\text { generación }\end{array}$ & $\begin{array}{c}\text { Peso de } \\
\text { racimo en } \\
1^{\text {ra }}\end{array}$ & $\begin{array}{c}\text { Peso de } \\
\text { mil } \\
\text { semillas }\end{array}$ & $\begin{array}{c}\text { Rendimiento } \\
\left(\mathrm{kg} \mathrm{ha}^{-1}\right)\end{array}$ \\
\hline REP & 3 & 29.45 & $54.01^{* *}$ & 0.45 & $1248.5^{* *}$ & 186.85 & 315931.5 & 727.8 & 860360.89 \\
Densidad & 1 & 4 & 1.89 & 20.25 & 805.1 & 81 & 2073240 & 15.01 & 25560.02 \\
Riegos & 1 & 9 & 3.51 & 3.06 & 9628.5 & 756.25 & 2148056 & 0.39 & $7740219.52^{* *}$ \\
Híbridos & 7 & $727.7^{* *}$ & $710.5^{* *}$ & $235.7^{* *}$ & $5844.42^{* *}$ & $1226.35^{* *}$ & 467260 & $36636.4^{* *}$ & $2011367.65^{* *}$ \\
Híbridos/ & 3 & 18.79 & 7.8 & 7.1 & 1512.55 & 69.58 & 460108 & 291.26 & 553311.14 \\
Densidad & & 3.19 & 2.8 & 1.2 & 11.7 & 10.37 & 19.36 & 4.01 & 20.6 \\
CV & & 0.95 & 0.94 & 0.92 & 0.77 & 0.82 & 0.48 & 0.95 & 0.65 \\
$\mathrm{R}^{2}$ & & & & & & & & & \\
\hline
\end{tabular}

Cuadrados medios y significancia, segunda fecha de siembra primavera-verano de 2016. 
Cuadro 9. Relaciones entre fuentes de variación y características de planta en el CEVAF.

\begin{tabular}{lcccccccc}
\hline Ciclo & $\begin{array}{c}\text { Días a } \\
\text { floración }\end{array}$ & $\begin{array}{c}\text { Fin de } \\
\text { floración }\end{array}$ & $\begin{array}{c}\text { Días a } \\
\text { madurez }\end{array}$ & $\begin{array}{c}\text { Altura } \\
\text { de } \\
\text { planta } \\
(\mathrm{cm})\end{array}$ & $\begin{array}{c}\text { Longitud } \\
\text { de racimo } \\
\text { en } 1^{\text {ra }} \\
\text { generación }\end{array}$ & $\begin{array}{c}\text { Peso de } \\
\text { racimo en } \\
1^{\text {ra }} \\
\text { generación }\end{array}$ & $\begin{array}{c}\text { Peso de } \\
\text { mil } \\
\text { semillas }\end{array}$ & $\begin{array}{c}\text { Rendimiento } \\
\left(\mathrm{kg} \mathrm{ha}^{-1}\right)\end{array}$ \\
\hline $1^{\text {ra }}$ FS & 68 & 83 & 143 & 233 & 64 & 2674 & 380 & 2473 \\
23000 & $67^{\mathrm{a}}$ & $83^{\mathrm{a}}$ & $141^{\mathrm{b}}$ & $230^{\mathrm{a}}$ & $63^{\mathrm{a}}$ & $2459^{\mathrm{a}}$ & $376^{\mathrm{b}}$ & $2464^{\mathrm{a}}$ \\
26000 & $67^{\mathrm{a}}$ & $82^{\mathrm{a}}$ & $143^{\mathrm{a}}$ & $237^{\mathrm{b}}$ & $63^{\mathrm{a}}$ & $2914^{\mathrm{b}}$ & $384^{\mathrm{a}}$ & $2482^{\mathrm{a}}$ \\
3 R. AUX & $67^{\mathrm{a}}$ & $83^{\mathrm{a}}$ & $141^{\mathrm{b}}$ & $216^{\mathrm{b}}$ & $59^{\mathrm{a}}$ & $2456^{\mathrm{b}}$ & $375^{\mathrm{b}}$ & $2137^{\mathrm{b}}$ \\
4 R. AUX & $67^{\mathrm{a}}$ & $82^{\mathrm{a}}$ & $143^{\mathrm{a}}$ & $252^{\mathrm{a}}$ & $68^{\mathrm{a}}$ & $2918^{\mathrm{a}}$ & $384^{\mathrm{a}}$ & $2850^{\mathrm{a}}$ \\
Promedio & 67 & 83 & 142 & 234 & 63 & 2684 & 380 & 2481 \\
\hline
\end{tabular}

Cuadrados medios y significancia, segunda fecha de siembra primavera-verano 2016.

En el Cuadro 10 se observa que los híbridos Maravilla, Chinatan y 2B-5 resultaron más rendidores que Maravilla y HB-8 para este ciclo primavera verano; sin embargo, no superó al testigo K-93 que mostro un rendimiento de $3924 \mathrm{~kg} \mathrm{ha}^{-1}$. No obstante, es necesario precisar que el excesivo crecimiento del hibrido Maravilla, tuvo dificultades para la cosecha manual, ya que la altura de recolección de la semilla, aspecto que fue indicado por Rodríguez y Duque (2010).

Cuadro 10. Comparación de medias de características morfológicas de planta e índice de selección de las variedades de higuerilla en el CEVAF.

\begin{tabular}{|c|c|c|c|c|c|c|c|c|}
\hline Híbrido & $\begin{array}{l}\text { Días a } \\
\text { floración }\end{array}$ & $\begin{array}{l}\text { Fin de } \\
\text { floración }\end{array}$ & $\begin{array}{c}\text { Días a } \\
\text { madurez }\end{array}$ & $\begin{array}{l}\text { Altura } \\
\text { de } \\
\text { planta } \\
(\mathrm{cm})\end{array}$ & $\begin{array}{l}\text { Longitud } \\
\text { de racimo } \\
\text { en } 1^{\text {ra }} \\
\text { generación }\end{array}$ & $\begin{array}{c}\text { Peso de } \\
\text { racimo en } \\
1^{\text {ra }} \\
\text { generación }\end{array}$ & $\begin{array}{l}\text { Peso de } \\
\text { mil } \\
\text { semillas }\end{array}$ & $\begin{array}{l}\text { Rendimiento } \\
\left(\mathrm{kg} \mathrm{ha}^{-1}\right)\end{array}$ \\
\hline Maravilla & $82^{a}$ & $98^{\mathrm{a}}$ & $151^{\mathrm{a}}$ & $270^{\mathrm{a}}$ & $45^{\mathrm{a}}$ & $2792^{\mathrm{a}}$ & $488^{a}$ & $1851^{\mathrm{a}}$ \\
\hline T. Eva & $70^{\mathrm{a}}$ & $79^{\mathrm{bc}}$ & $140^{\mathrm{ab}}$ & $136^{\mathrm{a}}$ & $58^{\mathrm{a}}$ & $953^{\mathrm{a}}$ & $341^{\mathrm{bc}}$ & $1799^{a}$ \\
\hline T. Zoya & $76^{\mathrm{a}}$ & $83^{a b c}$ & $130^{\mathrm{b}}$ & $93^{\mathrm{a}}$ & $43^{\mathrm{a}}$ & $1052^{\mathrm{a}}$ & $256^{\mathrm{c}}$ & $899^{a}$ \\
\hline T. Olga & $81^{\mathrm{a}}$ & $91^{\mathrm{ab}}$ & $130^{\mathrm{b}}$ & $113^{\mathrm{a}}$ & $41^{\mathrm{a}}$ & $1891^{\mathrm{a}}$ & $324^{\mathrm{bc}}$ & $1368^{\mathrm{a}}$ \\
\hline HB-8 & $62^{\mathrm{a}}$ & $78^{\mathrm{bc}}$ & $140^{\mathrm{ab}}$ & $241^{\mathrm{a}}$ & $74^{\mathrm{a}}$ & $2772^{a}$ & $339^{b c}$ & $2628^{a}$ \\
\hline T. K-93 & $65^{\mathrm{a}}$ & $71^{\mathrm{c}}$ & $130^{\mathrm{b}}$ & $139^{\mathrm{a}}$ & $48^{\mathrm{a}}$ & $2895^{\mathrm{a}}$ & $297^{\mathrm{bc}}$ & $3924^{\mathrm{a}}$ \\
\hline Chinatan & $64^{\mathrm{a}}$ & $79^{\mathrm{bc}}$ & $140^{\mathrm{ab}}$ & $230^{\mathrm{a}}$ & $69^{a}$ & $2679^{a}$ & $344^{\mathrm{bc}}$ & $2649^{a}$ \\
\hline 2-B5 & $60^{\mathrm{a}}$ & $76^{\mathrm{bc}}$ & $141^{\mathrm{ab}}$ & $241^{\mathrm{a}}$ & $69^{\mathrm{a}}$ & $2695^{\mathrm{a}}$ & $366^{\mathrm{b}}$ & $2850^{\mathrm{a}}$ \\
\hline Promedio & 70 & 82 & 137 & 183 & 56 & 2216 & 344 & 2246 \\
\hline
\end{tabular}

Segunda fecha de siembra primavera-verano de 2016.

Ello se considera una condición común para la mayoría de las plantas de higuerilla de porte medio a alto y aunque las variedades comerciales alcanzan entre 0.9 y $3 \mathrm{~m}$ (Flemming y Jongh, 2011), muchos productores prefieren las variedades enanas que crecen a una altura promedio de $1.6 \mathrm{~m}$, con períodos de siembra a cosecha de 120 a 130 días, así como las variedades medianas que crecen desde 2 a $2.5 \mathrm{~m}$, con períodos de siembra a cosecha de 150 a 240 días (Rodríguez y Duque, 2010), aspecto al que se le debe prestar atención. 


\section{Conclusiones}

El ciclo que más favorece el desarrollo del cultivo para HB-8 y 2B-5 es O-I con densidad de 23 000 plantas $\mathrm{ha}^{-1}$ y tres riegos de auxilio, para $\mathrm{P}-\mathrm{V}$ se recomienda cuatro riegos de auxilio, densidad de 23000 plantas ha $^{-1}$ y los híbridos de mejor respuesta fueron HB-8 y Chinatan.

Se concluye que R. communis estudiadas poseen características agronómicas que las diferencian entre sí, a la vez que demostraron la existencia de particularidades relevantes para la producción de aceite en función del contenido de sus semillas, la producción por planta y el estimado por unidad de área. Por ello se considera, R. communis representa una buena opción en términos de su empleo para la producción de aceite, el cual se puede destinar a la producción de biodiesel, así como otros derivados.

\section{Agradecimientos}

A la compañía Castor Fields, SA PI de CV, por facilitar los genotipos involucrados en esta evaluación.

\section{Literatura citada}

Amorim, N. M. da S.; Araujo, A. E. e de Beltrão, N. E. de M. 2001. Clima e solo. In: Pedrosa, de A. M.; Lima, F. E. e Ed, O. Agronegocio da mamona no Brasil. Embrapa Algodão, Campina Grande. 62-76 pp.

Beltrão, N. E. M. 2001. Fitologia. In: Azevedo, D. M. P. e Lima, E. L. (Eds.). O agronegócio da mamona no Brasil. Brasília: Embrapa Informação Tecnológica. 37-61 pp.

Cardona, A. C. A.; Orrego, A. C. E. and Gutiérrez, M. L. F. 2009. La higuerilla: una alternativa agroindustrial. Universidad Nacional de Colombia, sede Manizales. Ed. Artes Gráficas Tizán. 28(9):951-956.

Chan, A.; Crabtree, J.; Zhao, Q.;Lorenzi, H.; Orvis, J.; Puiu, D.; Melake-Berhan, A.; Jones, K.; Redman, J.; Chen, G.; Cahoon, E.; Gedil, M.; Stanke, M.; Haas, B.; Wortman, J.; FraserLiggett, C.; Ravel, J. and Rabinowicz, P. 2010. Draft genome sequence of the oilseed species Ricinuscommunis. Nature Biotechnol. 28(9):951-956

De Sousa-Schalick, G. D. R.; Peres, S. D.; Bottino, A. e Mazetti, F. 2011. Crecimento e produtivedade da mamona de porte baixo em diferentes espaçamentos e populações de plantas. Interciencia. 37(1):49-54.

Falasca, S. L.; Ulberich, A. C. and Ulberich, E. 2012. Developing an agro-climatic zoning model to determine potential production areas for castor bean (Ricinus communis L.). Industrial Crops and Products. 40:185-191.

FAOSTAT. 2011. Dirección de Estadística. http://faostat3.fao.org/home/S.

Flemming, N. and de Jongh, J. 2011. Castor (Ricinus communis). Potential of castor for bio-fuel production. FACT Project No. 146/WW/001. FACT Fundation. http://www.factfoundation.com/media_en/factsheet_castor.

Goytia-Jiménez, M. A.; Gallegos-Goytia, C. H. y Núñez-Colín, C. A. 2011. Relación entre variables climáticas con la morfología y contenido de aceite de semillas de higuerilla (Ricinus communis L.) de Chiapas. Rev. Chapingo Ser. Cienc. Forest. Amb. 17(1):41-48. 
Machado, C. G.; Martins, C. C.; Silva, L. B. and Cruz, S. C. C. 2009. Acta Scientiarum Agronomy. (31):293-299.

Machado, R.; Suárez, J. y Alfonso, M. 2012. Caracterización morfológica y agroproductiva de procedencias de Ricinus communis L. para la producción de aceite. Pastos y Forrajes. 35(4):381-392.

Moshkin, V. A. 1986a. Botanical and biological properties of castor. 11-64 pp.

Moshkin, V. A. and Alekseev, A. P. 1986b. Ripening of seeds. In: Moshkin, V. A. (Ed.). Castor. New Delhi, Amerind. 315 p.

Msaakpa, T. S. and Obasi, M. O. 2014. Corraled studies between growth and yield charaders of castro Bean (Ricunus communis L). int. J. Sci. Res. Publ. 4(7):1-10.

Pabón, G. G. 2010. Estudio de las características botánicas y etnobotánicas de higuerilla (Ricinus communis L.). Cultivos energéticos alternativos. Pontificia Universidad Católica del Ecuador. 9-23 pp.

Rafael-Rodríguez y Alfredo-Zamarripa, 2013. Competitividad de la higuerilla (Ricinus communis) para biocombustible en relación a los cultivos actuales en el estado de Oaxaca, México 1. http://ageconsearch.umn.edu/bitstream/143921/2/12\%20Higuerilla\% 20INIFAP_\%200AXACA.pdf.

Rico, P. H.; Tapia, V. L.; Teniente, O. R.; González, A. A.; Hernández, M.; Solís, B. J. y Zamarripa, C. A. 2011. Guía para cultivar higuerilla (Ricinus communis L.) en Michoacán. Boletín Técnico 1. INIFAP. 42 p.

Rivera-Brenes, P. A. y Hernández-Lopéz, J. 2016. Evaluación de rendimiento y calidad de aceite de siete de Ricinus communis. Universidad de Costa Rica Alajuela. Rev. Mesoam. 27(1):183-189.

Rodríguez, D. E. y Duque, J. S. 2010. Plan de negocios para el cultivo de higuerilla, estudio de caso. Municipio Balboa (Risaralda). Licenciado en Administrador Ambiental. Universidad Tecnológica de Pereira. Colombia. 100 p.

Rodríguez, H.; y Zamarripa, C. 2011. Competitividad de la higuerilla Ricinus communis para biocombustible en relación a los cultivos actuales en Oaxaca, México. Reunión Nacional del Innovación Agrícola núm. 6. 353 p.

Solís B. J. L.; Muñoz O. A.; Escalante E. J. A.S. y Zamarripa, C. A. 2011. Crecimiento de variedades y componentes del rendimiento de higuerilla (Ricinus communis L.) en Montecillo, Estado de México. Rev. Mex. Cienc. Agríc. 6(7):311-323.

Tavora, F. J. A. 1982 A cultura da mamona. Fortaleza. EPACE, Brasil. 111 p.

Toledo, B. R.; Coria, A. V. M.; Bautista, H. J. J.; Muñoz, F. H. J.; Vidales, F. J. A.; Castillo, V. J. C. y García, G. F. E. 2006. Experiencias en la producción de higuerilla Ricinus communis L. var.

Weiss, E. A. 1983. Oilseed crops. London. Longman. 660 p. 